\title{
Video Article \\ Dual Somatic Recordings from Gonadotropin-Releasing Hormone (GnRH) Neurons Identified by Green Fluorescent Protein (GFP) in Hypothalamic Slices
}

\author{
Peter J. Hemond ${ }^{1}$, Kelly J. Suter ${ }^{1}$ \\ ${ }^{1}$ Department of Biology, University of Texas San Antonio - UTSA \\ Correspondence to: Kelly J. Suter at kelly.suter@utsa.edu \\ URL: https://www.jove.com/video/1678 \\ DOI: doi:10.3791/1678
}

Keywords: Jove Neuroscience, Issue 36, electrophysiology, simultaneous recording, cell-attached recording, current clamp, brain slice

Date Published: 2/23/2010

Citation: Hemond, P.J., Suter, K.J. Dual Somatic Recordings from Gonadotropin-Releasing Hormone (GnRH) Neurons Identified by Green Fluorescent Protein (GFP) in Hypothalamic Slices. J. Vis. Exp. (36), e1678, doi:10.3791/1678 (2010).

\section{Abstract}

Gonadotropin-Releasing Hormone $(\mathrm{GnRH})$ is a small neuropeptide that regulates pituitary release of luteinizing hormone (LH) and folliclestimulating hormone (FSH). These gonadotropins are essential for the regulation of reproductive function. The $\mathrm{GnRH}$-containing neurons are distributed diffusely throughout the hypothalamus and project to the median eminence where they release $\mathrm{GnRH}$ from their axon terminals into the hypophysiotropic portal system (1). In the portal capillaries, $\mathrm{GnRH}$ travels to the anterior pituitary gland to stimulate release of gonadotropins into systemic circulation. GnRH release is not continuous but rather occurs in episodic pulses. It is well established that the intermittent manner of $\mathrm{GnRH}$ release is essential for reproduction $(2,3)$.

Coordination of activity of multiple $\mathrm{GnRH}$ neurons probably underlies $\mathrm{GnRH}$ pulses. Total peptide content in GnRH neurons is approximately $1.0 \mathrm{pg} / \mathrm{cell}$ (4), of which $30 \%$ likely comprises the releasable pool. Levels of $\mathrm{GnRH}$ during a pulse $(5,6)$, suggest multiple $\mathrm{GnRH}$ neurons are probably involved in neurosecretion. Likewise, single unit activity extracted from hypothalamic multi-unit recordings during LH release indicates changes in activity of multiple neurons (7). The electrodes with recorded activity during LH pulses are associated with either GnRH somata or fibers (8). Therefore, at least some of this activity arises from GnRH neurons.

The mechanisms that result in synchronized firing in hypothalamic $\mathrm{GnRH}$ neurons are unknown. Elucidating the mechanisms that coordinate firing in $\mathrm{GnRH}$ neurons is a complex problem. First, the $\mathrm{GnRH}$ neurons are relatively few in number. In rodents, there are $800-2500 \mathrm{GnRH}$ neurons. It is not clear that all $\mathrm{GnRH}$ neurons are involved in episodic $\mathrm{GnRH}$ release. Moreover, $\mathrm{GnRH}$ neurons are diffusely distributed (1). This has complicated our understanding of coordination of firing and has made many technical approaches intractable. We have optimized loose cell-attached recordings in current-clamp mode for the direct detection of action potentials and developed a recording approach that allows for simultaneous recordings from pairs of $\mathrm{GnRH}$ neurons.

\section{Video Link}

The video component of this article can be found at https://www.jove.com/video/1678/

\section{Protocol}

1. Hypothalamic brain slices are prepared, incubated and transferred to the recording chamber as previously detailed (9) in animals whose $\mathrm{GnRH}$ neurons express green fluorescent protein (GFP) under the control of the GnRH peptide promoter (10). After removal of the brain from the animal, the brain is blocked using a razor blade to eliminate regions that are not of interest. In order to determine the regions to remove, the brain is placed on its dorsal surface so that the hypothalamus can be visualized. For both the coronal and sagittal slice orientations, the cerebellum is removed. For coronal slice preparation, the rostral portion of the cortex is removed. In this slice orientation, the lateral regions of the cortex provide support for slicing the brain and provide sufficient area of brain for the placement of silver wires to secure the brain in the recording chamber. For the sagittal slice preparation, the lateral regions of the brain are removed and the rostral portions of the cortex provide the support for brain slicing and for placement of silver wires to secure the brain in the recording well. Throughout the blocking procedure, the brain is continuously moistened with cold artificial cerebrospinal fluid (ACSF) using a glass pipette.

2. A small amount of Superglue is placed on the cutting platform. The brain is lifted with a spatula. Excess ACSF is removed by bring a Kimwipe to the surface of the spatula and drawing excess ACSF away from the brain. The brain is gently slid forward onto the glue. It is important to not to touch the brain or push down on it in order to secure it. The weight of the brain itself is usually sufficient to ensure it is secure.

3. The brain and the cutting platform is then secured to the vibrating microtome and the cutting well is filled with cold ACSF. The cold solution both firms the brain and may protect from damage during the cutting procedure. Different brain regions vary in the degree of coldness that optimizes quality slices. The temperature of the ACSF for preparation of hypothalamic slices is generally $0^{\circ} \mathrm{C}$.

4. Slices should be cut slowly and each slice should float freely away from the brain. If slices begin to curl during cutting, the speed at which the blade is advancing should be slowed. One can use a small paint brush to uncurl slices but this does risk damaging the slices and thus, should be avoided. Slowly the speed of advance of the blade is preferred over using a paint brush. 
5. As each slice is cut from the brain, it is withdrawn from the cutting chamber and placed in a slice incubation chamber in a warm water bath (approximately $32^{\circ} \mathrm{C}$ ). The slice incubator is continuously supplied with oxygen using a mixture of $95 \% \mathrm{O}_{2}$ and $5 \% \mathrm{CO}_{2}$. Generally, prerecording slice incubation ranges from 30 minutes to 2 hours.

6. Glass pipettes (3-6 M $)$ are fabricated as previously described (9) using a vertical puller. The temperature and duration of pulling varies based on the type of pipette puller one uses, the type of glass one uses and the lifetime of the heating filament of the puller. The shape of the pipette tip can also vary depending on the user's preference. We have achieved good success with a uniformly tapered tip with an opening of $0.1 \mathrm{~mm}$. Larger openings in the pipette tend to damage the neurons while smaller openings fail to result in long duration recordings.

7. Pipettes coated with Sylgard to reduce capacitance. Pulled pipette tips are lightly coated with Sylgard 184 using a second pipette. Sylgarded tips are then heat-cured using a heat gun. These pipettes are then filled with the bath perfusate. To increase visualization of the pipette tips, a small amount of Alexa-568 is added to the portion of the bath solution to be used as the pipette solution before filling the pipettes. We do not weigh the Alexa-568 but rather use enough to turn the solution pink by eye. The pipette tips can then be visualized under the Texas red filter.

8. We have developed an approach to obtain current-clamp recordings for detection of action potentials using low resistance seals (see Discussion). Recordings are performed at the cell's endogenous resting potential with no applied current using Axon Instrument's $2 \mathrm{~B}$ Axoclamp. However, due to the low resistance seal, one could not detect action potentials with just the $2 \mathrm{~B}$ amplifier. To increase the signal, a second amplifier (AM Systems 3000) is used in series with the Axoclamp 2B. This approach has the ease of loose seals and utility of long-term recordings. Technically, one can directly measure action potentials, thereby circumventing the problems with the approach in the voltage-clamp recording mode (see Discussion).

9. One takes two pipettes to the surface of the two previously selected neurons at the same time, in a similar fashion as the approach for the whole cell recording configuration. Approaching both neurons at the same time has been more successful in obtaining the dual recordings than attempting each neuron of the pair independently. During this time, current injection pulses are passed from the $2 \mathrm{~B}$ amplifier as is done during the approach for whole-cell recordings.

10. Positive pressure must be maintained on both pipettes to prevent clogging the tip. Positive pressure for dual recordings is best generated by using an unfilled $3 \mathrm{ml}$ empty syringe attached to the pipette holder by a length of piece of tubing. Using syringes allows one to maintain pressure on both pipettes. Positive pressure should be applied as soon as the pipette has been placed in the bath solution of the recording chamber. One approaches the selected neurons with the pipettes sequentially. After positioning the first pipette directly over the largest surface of the neuron, one maintains positive by leaving the syringe in place and leaves the pipette in this position. One then returns to the top of the recording chamber and lowers the second recording pipette into position. Selected neurons are often at different levels of the slice. The first pipette should be positioned over the neuron that is deepest in the slice and position the second pipette at the more superficial neuron. This prevents the positioning of the second pipette from shifting the positioning of the second pipette as the slice will move slightly in the vertical plane as pipettes enter the slice.

11. Loose seals (18-30 M $\Omega$ ) will be used. The positive pressure will induce a small dimple on the surface of each cell. One attempts to seal the first neuron by releasing positive pressure and applying very slight negative pressure. The positive pressure is released by removing the syringe. Suction by mouth is then applied to the free end of the tubing. A healthy neuron's membrane will quickly respond to the release of positive pressure and adhere to the pipette. Slight suction will form a seal with appropriate resistance in a healthy neuron.

12. When one attempts to seal a neuron and fails, the pipette cannot be re-used since membranes only seal to very clean glass. Instead, one raises the pipette (and microscope objective) away from the surface of the slice to the top of the perfusion well, removes the pipette from the bath, changes to a clean pipette and then returns to the slice to attempt to seal another cell. For these reason, it is important to select multiple potential neurons for recordings. For slices with less than 4-6 recording quality neurons from which to choose, the success in obtaining dual recordings is limited.

13. After establishing a loose seal based on resistance, the current injection pulses from the $2 \mathrm{~B}$ amplifier are terminated. The $2 \mathrm{~B}$ amplifer is placed in series with the 3000 series amplifer. In this configuration, the output of the $2 \mathrm{~B}$ amplifer serves as the input for the 3000 series amplifer. The latter amplifer provides signal for data acquisition.

14. The seal (step 4) and reconfiguration of amplifers (step 5) is then repeated for the second neuron.

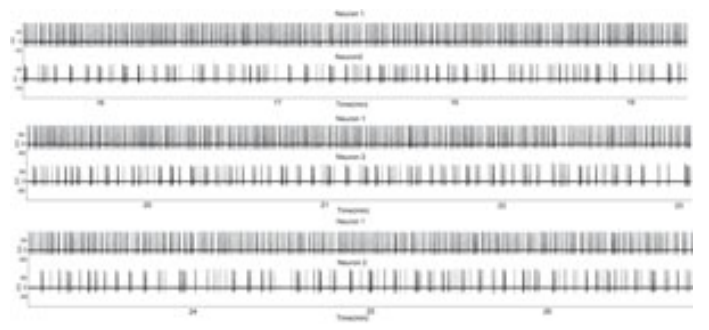

Figure 1. A period of increased firing in two GnRH neurons using the loose seal attached recording configuration in a sagittal slice preparation. The slice was derived from a castrated male. Each upward deflection indicates an action potential. Note that one GnRH neuron (top traces) exhibits nearly continuous firing while the second $\mathrm{GnRH}$ neuron exhibits intermittent bursts. This pattern of activity from single $\mathrm{GnRH}$ neurons is similar to that of single units extracted from multi-unit recordings during hormone secretion in vivo (7). Please click here to see a larger version of figure 1.

\section{Discussion}

The activity of interest in some neurons including GnRH neurons (based on hormone secretion) occurs on time scales of hours (5-7). Therefore, the whole cell configuration is not the best choice for some experimental goals due to the dialysis of intracellular messengers in the whole cell recording mode. Secondarily, whole-cell recordings are generally limited to animals less than about 120 days of age. With age, neuronal membranes appear to stiffen, making high resistance seals difficult to achieve. Additionally, if one obtains a high resistance seal, rupturing the patch disrupts the seal, leaving a hole between the pipette and the membrane. This leads to an unusable recording and a neuron that will quickly die due to ionic imbalances. Regular ovarian cycles, and thus, stable activity of the GnRH pulse generator does not occur until later in life (7-10 months of age in C57BI6 females; 11, 12), beyond the age when one can reasonably anticipate obtaining whole-cell recordings reliably. 
Finally, whole-cell recordings destroy the endogenous ratios of internal and external ion concentrations. With whole cell recordings, the internal concentration of any ion equals the concentration of the ion in the pipette solution. This is because the pipette solution's relatively large volume rapidly reaches equilibrium with/replaces the relatively small endogenous volume of the cell.

The loose cell attached approach circumvents many of the limitations of whole-cell recordings. First, a low resistance seal (15-30 $\mathrm{M} \Omega$ ) can be used. These are relatively easy to form even in neurons from older animals. Secondly, one does not rupture the sealed patch of membrane. Therefore, loose cell attached recordings are technically much easier than whole-cell recordings. Additionally, since the cell membrane is intact, dialysis of intracellular components does not occur and endogenous ionic ratios are preserved. One can not use the loose cell attached approach for studying synaptic currents but it is ideal for long-term recordings from neurons in a relatively non-invasive manner. The cellattached recordings can also be performed using any standard intracellular solution in the pipette. This offers the additional advantage of rupturing the membrane patch when the long term recording is completed and labeling the neuron with an intracellular marker.

The loose cell attached approach has been used in the voltage-clamp recording mode. However, voltage-clamp recording in the loose cell attached configuration has several methodological problems. First, the recorded signal is an indirect measure of activity. The signal that is measured (as the so-called action current) is the capacitive current that charges the membrane (13). This is an extremely important methodological issue. The capacitance and resistance of a recording pipette can filter the recorded signal. It is very likely that the small action currents are lost in charging the capacitance of the pipette which, cannot be properly compensated with most amplifiers, due to the high resistance of the headstage. When these signals go undetected, the apparent firing pattern of the neuron does not reflect the true firing pattern. Likewise, uncompensated pipette and seal resistances cause significant errors in measurements during changes such as when action currents are expressed (13). Some amplifiers provide capacitance and resistance "compensation" for the pipette and seal, which limits signal loss, but high resistance head stages of most amplifiers hinder optimal compensation. Secondly, an artificial situation is imposed on the cell. In voltageclamp mode, the area around the cell membrane is held to a fixed potential, in these studies, $0 \mathrm{mV}$. This does not mean there is no current applied to the cell membrane. The signal measured in voltage-clamp is actually the amount of current applied to the membrane to maintain the fixed potential. Therefore, this applied current can alter the cell activity.

Dual recordings in the $\mathrm{GnRH}$ system are particularly challenging due to the limited number of GnRH neurons and their diffuse distribution. For dual recordings to be successful, the manipulator must be exceedingly stable. Even slight movement of the electrode can cause the pipette to slip off the neuron and end the recording. Moreover, movement of the pipette on the cell (e.g., re-positioning to compensate for movement) can alter firing patterns. Some ion channels such $\mathrm{N}$-type calcium channels are mechanically sensitive: membrane stretch causes repetitive activity in both whole-cell and cell-attached recording configurations (14). Finally, the manipulator system must be capable of exceedingly fine and smooth motion. As noted above, with dual recordings, one takes two pipettes to the surface of the two previously selected neurons at the same time and attempts to seal one neuron. If successful, then one attempts to seal the second cell. Generally, one cannot expect to seal and have a high quality recording with every attempt. This, however, creates a particular problem with dual recordings. If one is successful with the first neuron but fails with the second, one must change the pipette and try a different cell. Therefore, one must be able to move both the immersion objective of the microscope and the pipette to the top of the perfusion well (to change the pipette) without disrupting the successfully sealed neuron.

Our development and use of the loose cell-attached approach for dual recordings is a major technical advance in studying $\mathrm{GnRH}$ neurons. It is likely to produce useful results that will help move the field forward in the context of the critical question of what mechanisms underlie the coordinated activity that results in pulsatile hormone secretion.

\section{Acknowledgements}

I am grateful to Ronald L. Calabrese, Dieter Jaeger (Emory University) and Ward Yuhas (Axon Instruments) for useful technical discussions.

\section{References}

1. Silverman, A.J. The gonadotropin releasing-hormone (GnRH) neuronal systems: immunocytochemistry. In: Knobil E and Neill JD (eds). The Physiology of Reproduction. Raven Press, New York (1994).

2. Freeman, M.E. The neuroendocrine control of the ovarian cycle of the rat. In: The physiology of reproduction. In: Knobil E and Neill JD (eds). The Physiology of Reproduction. Raven Press, New York (1994).

3. Belchetz, P.E., Plant, T.M., Nakai, Y., Keogh, E.J., \& Knobil, E. Hypophysial responses to continuous and intermittent delivery of hypopthalamic gonadotropin-releasing hormone. Science 202, 631-633 (1978).

4. Maurer, J.A., \& Wray, S. Luteinizing hormone-releasing hormone quantified in tissues and slice explant cultures of postnatal rat hypothalami. Endocrinology 140, 791-799 (1999).

5. Harris, G.C. \& Levine, J.E. Pubertal acceleration of pulsatile gonadotropin-releasing hormone release in male rats as revealed by microdialysis. Endocrinology 14, 163-171 (2003).

6. Sisk, C.L., Richardson, H.N., Chappell, P.E., \& Levine, J.E. In vivo gonadotropin-releasing hormone secretion in female rats during peripubertal development and on proestrus. Endocrinology 142, 2929-2936 (2001).

7. Cardenas, H., Ordog, T., O'Byrne, K.T., \& Knobil, E. Single unit components of the hypothalamic multiunit electrical activity associated with the central signal generator that directs the pulsatile secretion of gonadotropic hormones. Proc Natl Acad Sci U S A. 90, 9630-9634 (1993).

8. Silverman, A.J., Wilson, R., Kesner J.S., \& Knobil, E. Hypothalamic localization of multiunit electrical activity associated with pulsatile LH release in the rhesus monkey. Neuroendocrinology 44, 168-171 (1986).

9. Roberts, C.B., O'Boyle, M. P., \& Suter, K.J. Dendrites determine the contribution of after depolarization potentials (ADPs) to generation of repetitive action potentials in hypothalamic gonadotropin releasing-hormone (GnRH) neurons. J Comput Neurosci. 26, 39-53 (2009).

10. Spergel, D.J., Kruth, U., Hanley, D.F., Sprengel, R., \& Seeburg, P.H. GABA- and glutamate-activated channels in green fluorescent proteintagged gonadotropin-releasing hormone neurons in transgenic mice. J Neurosci. 19, 2037-2050 (1999).

11. Nelson, J.F., Felicio, L.S., Randall, P.K., Sims, C., \& Finch, C.E. A longitudinal study of estrous cyclicity in aging C57BL/6J mice: I. Cycle frequency, length and vaginal cytology. Biol Reprod. 27, 327-339 (1982). 
12. Gee, D.M., Flurkey K., \& Finch, C.E. Aging and the regulation of luteinizing hormone in C57BL/6J mice: impaired elevations after ovariectomy and spontaneous elevations at advanced ages. Biol Reprod. 28, 598-607 (1983).

13. Anson, B.D., \& Roberts, W.M. Loose-patch voltage-clamp technique. In: Patch-clamp analysis: Advanced techniques. Edited by: W Walz, AA Boulton and GB Baker. Neuromethods 35; Humana Press. Pgs 265-286 (2002).

14. Calabrese, B., Tabarean T., Juranka, P., \& Morris, C.E. Mechanosensitivity of N-type calcium channel currents. Biophys J. $83,2560-2574$ (2002).

15. Higure, Y., Katayama, Y., Takeuchi, K., Ohtubo, Y., Yoshii, K. Rapid killing of single neurons by irradiation of intracellularly injected dye. Science 206, 702-704 (1979).

16. Higure, Y., Katayama, Y., Takeuchi, K., Ohtubo, Y., Yoshii, K. Lucifer Yellow slows voltage-gated Na+ current inactivation in a light-dependent manner in mice. J Physiol 550, 159-167 (2003). 